\title{
Improving Agricultural Production using Internet of Things (IOT) and Open Source Technologies
}

\author{
Benjamin Odoi-Lartey \\ Kwame Nkrumah University of Science and \\ Technology \\ Computer Science Department \\ PMB University Post Office, Kumasi, Ghana
}

\author{
Edward Danso Ansong \\ Kwame Nkrumah University of Science and \\ Technology \\ Computer Science Department \\ PMB University Post Office, Kumasi, Ghana
}

\begin{abstract}
This article proposes a sensor network framework built using Internet of Thing (IoT) and Opens Source tools to automate farm fields monitoring in order to have the farm communicate real time with the necessary stakeholders. The study reviewed a couple of Internet of Things technology in Agriculture and used Open source software and hardware technologies to build-up a prototype that is cheaper and easy to deploy compared to existing proprietary systems. The designed system uses Raspberry Pi model 3 that serves as base station for the sensor network and connects with an Arduino Uno serving as an intermediary between various sensors and the Raspberry Pi. Among the sensors integrated in the system are DHT11 Soil Sensor, Humidity and Temperature Sensor, Passive Infra-Red (PIR) Sensor and Raspberry Pi Camera Module. The study deployed the prototype and tested it over Open IoT API platforms like KAA and OpenIOT for automation, monitoring and data collection
\end{abstract}

\section{Keywords}

Internet of Things, IoT, Raspberry Pi, Arduino, Sensors, iFarm, Open Source, Agriculture.

\section{INTRODUCTION}

The Internet has been the greatest invention in the $21 \mathrm{st}$ century improving various facets of life and advancing society in health, education, transportation, and communication. Agriculture is no exception from these improvements so far as the Internet is concerned [8].

Many primary experts in the field emphasised agriculture due to abundant resources associated with it and its ability to transfer surpluses to the more important industrial sector. Stringer [15] stated that "the primary role of Agriculture in the transformation of a developing economy was seen as subordinate to the central strategy of accelerating the pace of industrialisation" [15] [16].

Enu [2] postulates that field of agriculture still remains one of the most essential sectors for the economies of most countries across the globe. Ghana for instance has Agriculture contributing about $40 \%$ to its gross domestic product (GDP).

Research has shown that the focus of earlier works to improve Agriculture has been with the mechanical machineries and improvement of products such as varieties of seedlings and breeds of animals [6].

The Internet of things has taken over the world and businesses that seek to integrate it in their setup are usually on top of their competitors or predicted to be on top in the coming years. [12]

\section{LITERATURE REVIEW}

This article presents the literature review on IoT and more specifically its implementation in the agricultural sector. It provides an overview of previous research on the use of technology in Agriculture specifically Internet of Things technologies. It looks at a couple of Internet of Things technologies that are been researched into and currently making agriculture less difficult and increasing farm yield.

Within less than half a decade, much has been researched into so far as IoT in the field of Agriculture is concerned. It is very essential to set the context of the literature review work by looking into previous research works by authors in the field of sensor networks, sensor boards, data monitoring, security of IoT and finally reviewing on the field of IoT in Agriculture.

IoT is consistently developing and is a hot research point where openings are unending. Creative impulses are vast which have put it nearly reshaping the present type of web into a changed and coordinated rendition. With the persistent headways in innovation this potential IoT development is descending the street which is prospering as a pervasive worldwide figuring system where everybody and everything will be associated with the Web [5].

New inventive Internet of Things systems are inclining to issues of Agriculture production and escalating the worth, supportability and cost effectiveness of rural agricultural. The wide-ranging ranches we have today can, for example, use these Internet of Things technologies to screen sensors that can identify soil fertility and nutrients readiness remotely to help remotely oversee and control their reaping and water system hardware, and use computerized reasoning based investigation to rapidly break down operational information consolidated with outsider data, for example, climate administrations, to give new bits of knowledge and enhance decision making [17].

According to FAO [3] [4], World population is estimated to increase by $33 \%$ in the year 2050 and rural Agricultural production will have to increase by $60 \%$ to feed this population. Farming must along these lines change itself on the off chance that it is to bolster a developing worldwide populace and give the premise to financial development and neediness lessening. Environmental change will make this assignment more troublesome under the same old thing situation, because of unfavorable impacts on horticulture, requiring spiraling adjustment and related expenses.

FuBing [7] proposed a system structure based on Internet of Things that integrates wireless sensor network, $4 \mathrm{G}$ network and the Internet to realize the farmland air and soil environment data collection and analysis. The proposed 
system design has three layers thus the farmland environment information collection layer, the network transport layer and the system application layer. In between the network layer and the sensory layer are Mobile devices that are used to collect data such as photographs, and description voices and transmitted to the cloud platform with accessing Internet by $4 \mathrm{G}$ wireless communication network.

Ma et al [10] report in their paper that via sensory nodes, agriculture and other fields would be connected to Internet of Things thus allowing us to establish a common relation among agronomists, farmers farm fields and other stake holders irrespective of their geographical locations and this will aid the stake holders to have a better considerate of crop growth models and improve on methodologies in farming.

Ma et al [10] made a comparison between two different available boards thus MSP430F1611 and ATmega1281/AT86RF230. Out of these boards this article considered using an open version of similar boards to build another architecture called the iFarm Architecture.

\subsection{IoT APIs (Application Programming Interfaces)}

Jayaraman and Palmer [9] in their research proposed a semantically improved digital agriculture use case Phenonet developed with OpenloT platform (Phenonet-OpenIoT). The platform demonstrated how the semantic interoperability of the OpenloT platform helps in addressing the challenges posed by Phenonet applications. Their work extended the OpenloT stage to bolster the Phenonet utilize case by building up an area particular Phenonet philosophy in view of W3C SSN standards to connection sensor assets with space ideas and joining novel semantic thinking capacities and future work incorporates augmenting the utilization of OpenloT stage to more assorted administrations in computerized farming.

Among other Open Source platform that this journal paper revealed included but not limited to; Kaa, ThingsBoard, OpenIoT, Predix, Oracle Internet of Things, Carriot, SalesForce, Cisco IoT Platform, ThingWorx etc.

\subsection{Review of Related Architecture}

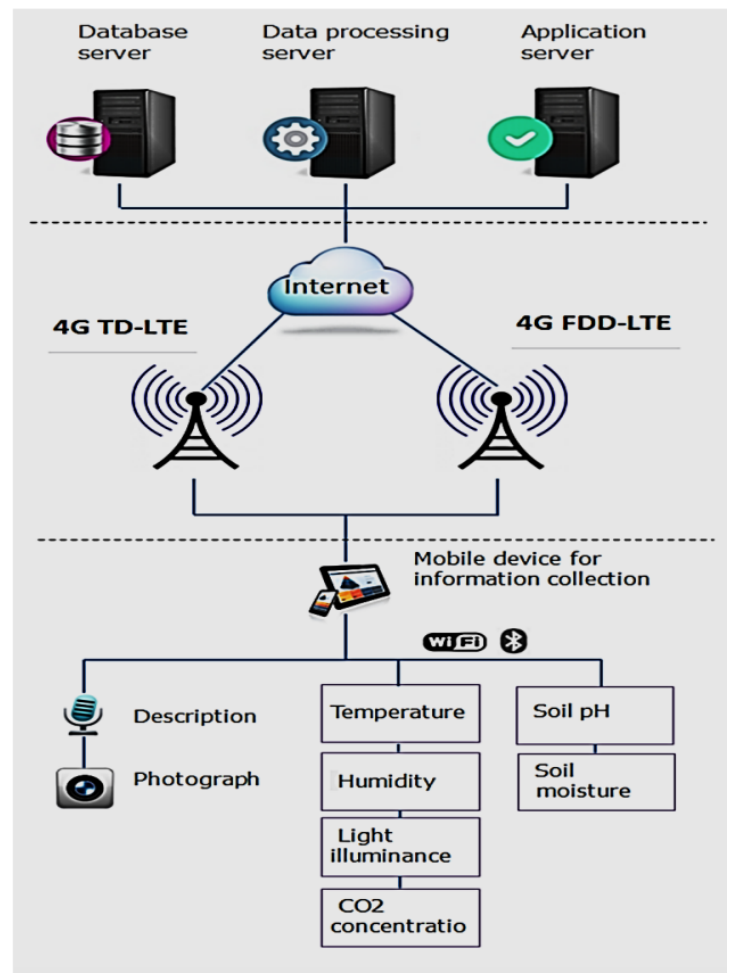

Fig 1: Fubing Architecture [7]

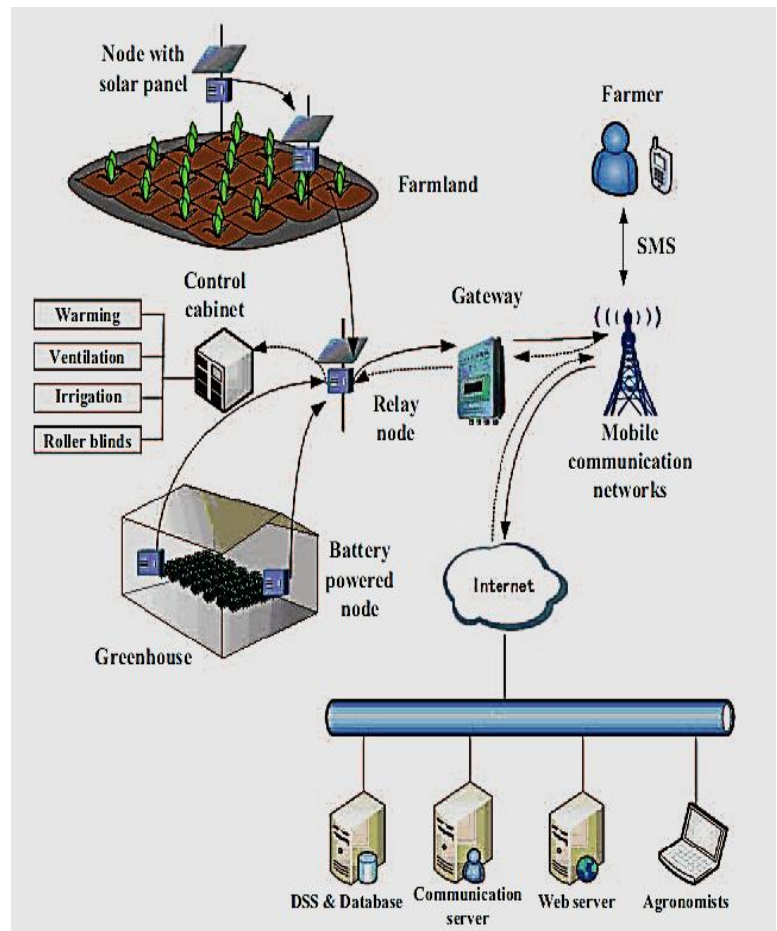

Fig 2: Ma et al Architecture [10]

\section{METHODOLOGY - DESIGN AND PROTOTYPE BUILDUP}

According to Rankin and Rankin any agriculture technology should address practical problems that are agreed upon by farmers and technology providers and the technology should fit into the five criteria for judging sustainable agricultural technology [13]. 
The five criteria for judging sustainable agricultural technology includes; Localization, Holistic, Usefulness, Affordability and Democratic. They research adopted the IDEO Human-centered Design approach as a guideline for the Prototype buildup.

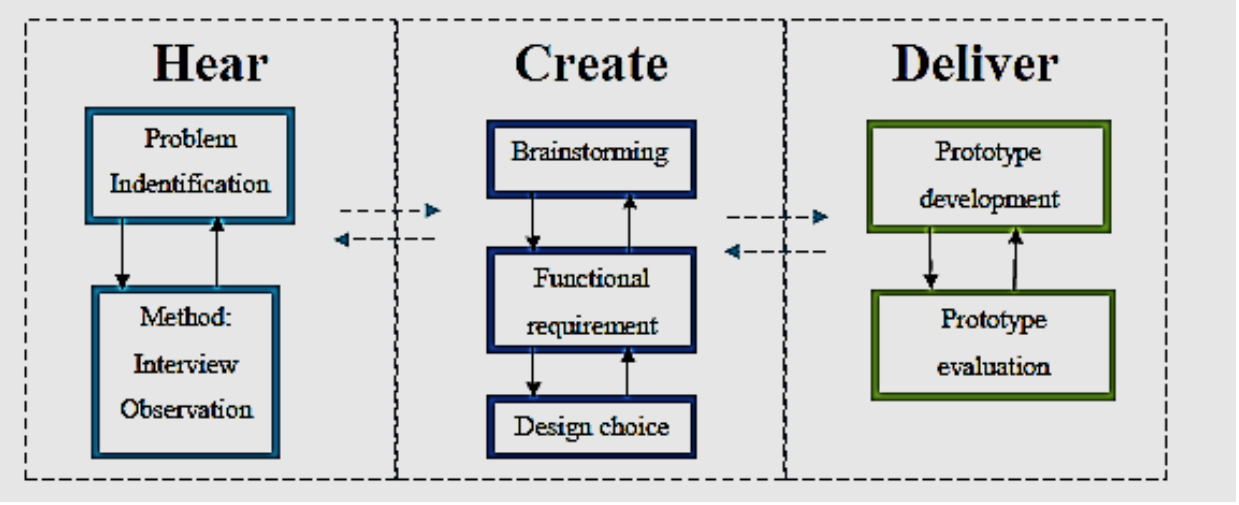

Fig 3: IDEO Human-Centered Design Process

\subsection{Schematics for the various Sensing Modules}

\section{Soils Sensor Schematic}

Aronson [1] in his Farmbot white paper shows soil sensor has three key connectors that links it to the Arduino Microcontroller. It has a VCC for voltage with maximum volts of $5 \mathrm{~V}$ with another connector for ground and finally a single connection for data transmission. The connector for data or signal transmission can be Digital or analog and the Arduino microcontroller has connection for all the two. The Arduino is programmed using Arduino IDE Software. The program is uploaded and output is displayed on the Console.

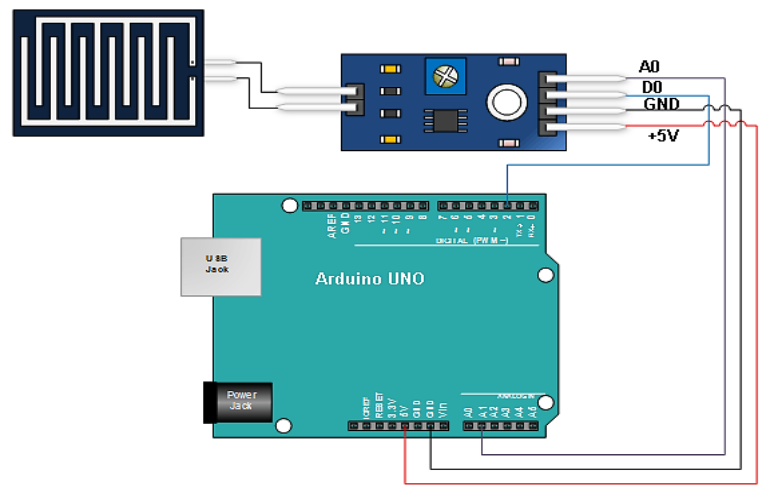

Fig 4: Soil Sensor Schematic

\section{Temperature/Humidity Module (DHT11)}

The DHT11 sensor has four connectors out of which three links to the Arduino microcontroller for sensed data interpretation and communication as shown in Figure 3.18. It has a VCC for voltage with maximum volts of $5 \mathrm{~V}$, another for ground and finally a single connection for data transmission. The connector for data or signal transmission can be Digital or analog and per the choice made, the Arduino will work interpret accordingly. The program is uploaded and output is displayed on the Console or pushed to the cloud API platform for remote access.

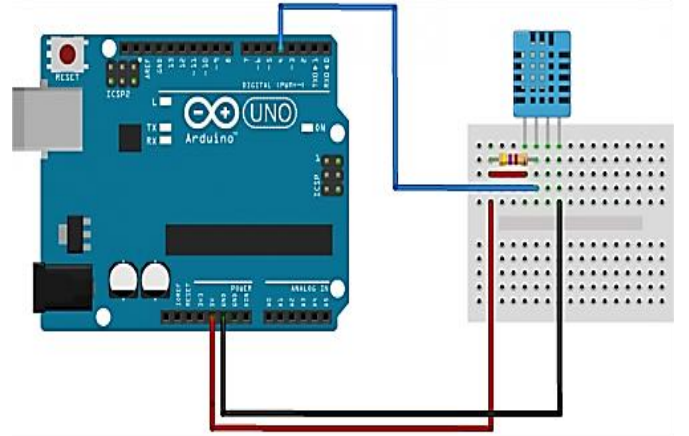

Fig 5: Humidity and Temperature Sensor Schematic

\section{Motion Detection and Image Capture}

Module: the motion detection and Image Capture module is designed on the Raspberry Pi 3 system on a chip (SOC) board which has the capabilities of both embedded systems and a full Input/output computer system. A motion detection sensor is interfaced with the GPIO of the Raspberry Pi and the camera module is connected to the normal display connector for Camera module. The logic behind is simplified caption of the camera anytime an unusual image is seen on the field. Aside that, timed captions are made from the day one of setup through to harvesting and post harvesting.

The various module can work independently on their own but integrating these modules brings about a more advance system that works cohesively producing a better results for field monitoring and accumulating field data over a period of time. The system is designed by integrating these various modules to have a more advance system. To implement the system there is the need to consider each farmers preference. [11] 


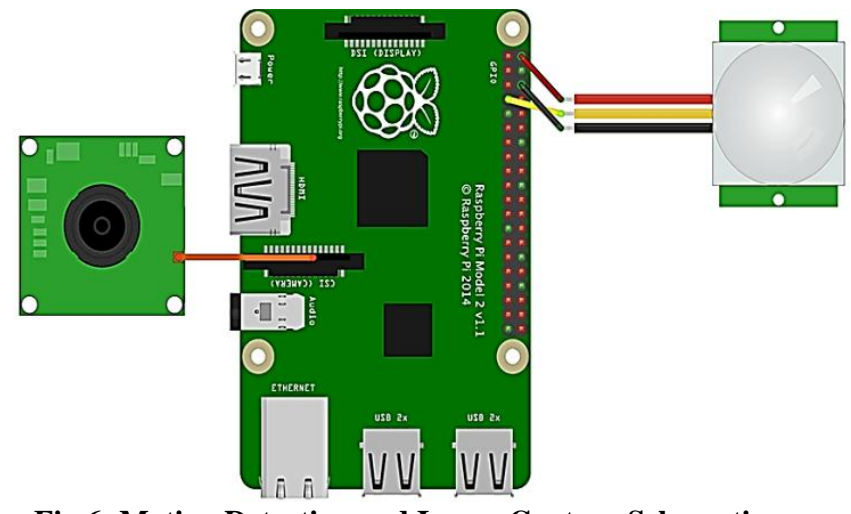

Fig 6: Motion Detection and Image Capture Schematic

\subsection{Overview and Architecture of the iFarm Architecture}

The proposed system is made up of both open source hardware and software components as shown in Figure 3.2. Out of the brainstorming with the experts on the field and identified quality attributes shown in Table 3.1: System quality attributes. The bottom most layer of the architecture has the various sensor that are connected to the base station (BS) which comprises Raspberry Pi Arduino and GSM Module. The BS is interfaced with the cloud through services provided by telecommunication companies for Internet connectivity sake since the third party cloud service run on the Internet. Among sample API platforms chosen as testbed KAA, Allseen Alliance and OpenIoT which sits in the cloud as both application and database server to the user [14]

\section{System Architecture Overview}

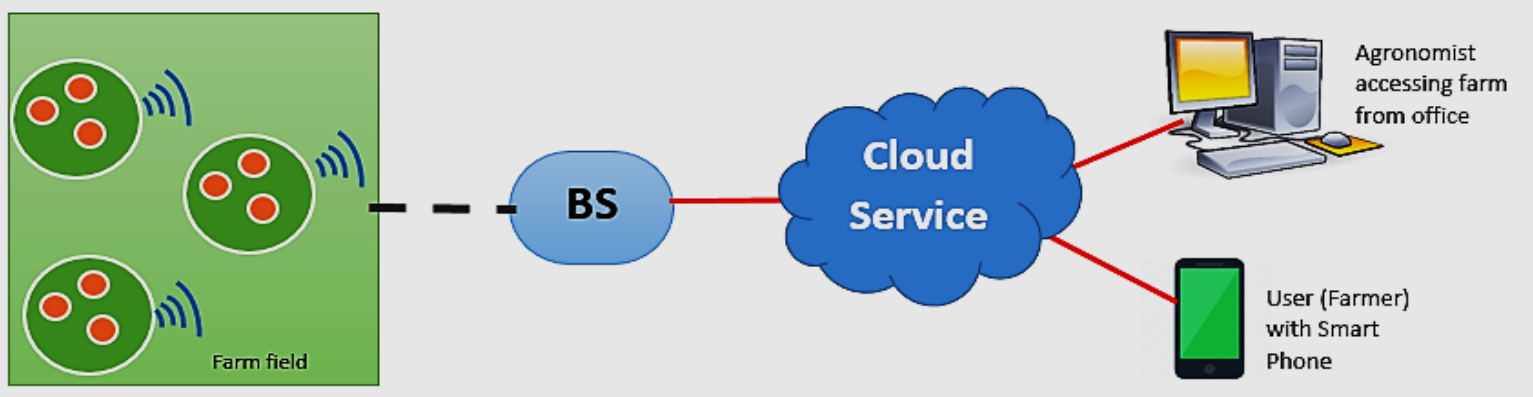

Fig 7: System Architecture Overview

\subsection{Sub layers of the iFarm Architecture Sensory Layer}

The bottommost layer is called the Sensory Layer which is basically made up of collection of sensors that reads sensory data from the farm fields and get it logged to the base station. By automation, data is generated at periodic intervals as the system has been programmed. The generation of the data occurs per assigned periods of activeness meaning when the nodes are not in active mode, they are deemed to be asleep or in a passive mode.

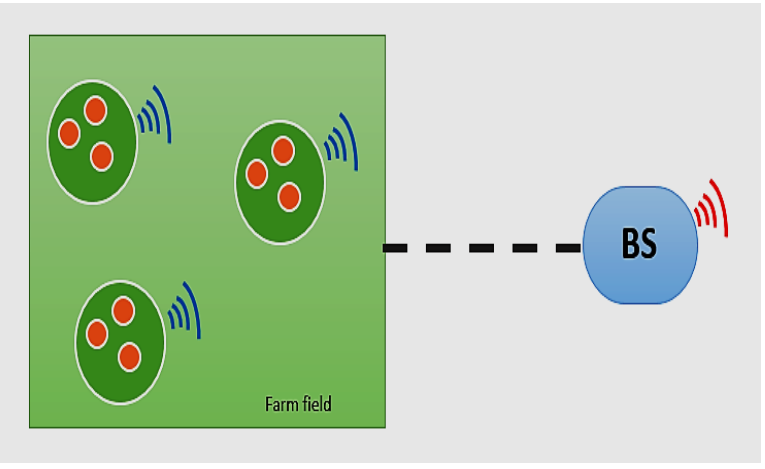

Fig 8: Sensing Unit

Among the sensors used in the prototype build up are Soil Sensor, Humidity/Temperature Sensor (DHT11), and Motion Sensor etc. A collection of these sensors based on farmers' preference makes up the sensory subsystem. It consist of tiny sensors which senses the changes of voltages and feeds the changes of measured voltage to an ADC (Analog to Digital Converter), the digital converted signals is sent to the CPU (Central Processing Unit) for further processing. The sensing subsystem in the proposed prototype here includes, Camera module, Soil sensor, Humidity and temperature sensor. The sensory layer interfaces with a processing subsystem which has an external memory subsystem and gives the system the capability of reading and storing data onsite.

\section{Network Layer}

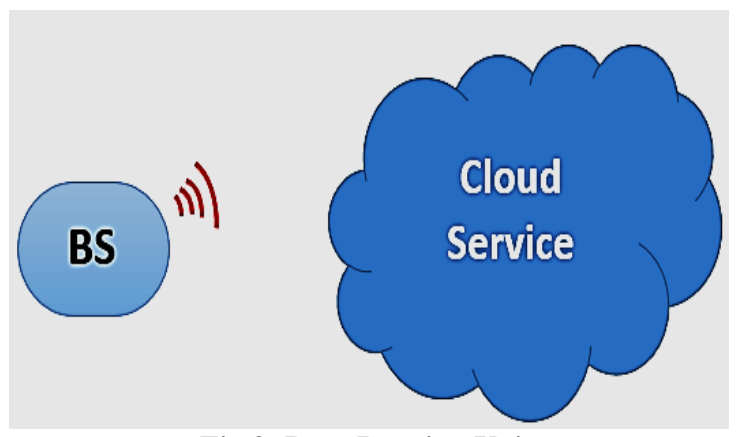

Fig 9: Data Logging Unit

The GSM/GPRS module serves the purpose of creating connectivity to the Internet and giving GPRS functionalities to the system. It has a transceiver having a wireless antenna, responsible for transmitting the radio signals using the optical signals or infrared waves as a medium, operates in ISM 
(Industrial, Medical and Medical purposes) band. It uses a free radio frequencies for communication in most countries, allocated in the international table of frequency allocation, contained in Article s5 of the radio regulations (volume 1). (Wireless Communication, Jean Paul Linnartz Reference website). The Data Logging unit is built around the Network layer and as such giving it the capability to easily log data to the online repository for further analysis. This unit comprises Arduino and Raspberry Pi and the main Network capability is dependent on the GSM module as it provides network connectivity to the raspberry pi.

\section{Application Layer}

The system is designed with the application layer in mind to make data access easier and readily available for users. The uploaded sensor data sits on the cloud and users can access the data through personal computers or smart phones with internet connectivity. In order to make data presentation so meaningful to users, the sensor readings should be displayed with visualizations like graphs to give a brief or summary of data at a glance. The GSM/GPRS module attached to the system makes location of each base station (BS) easily identified with base coordinates. Per the specified time interval programmed on base station, sensor readings are continually uploaded to the cloud platform and this information is made available for access from any web browser with Internet connectivity. The readings can always be read numerically or graphically through the API dashboard at the user end. The readings are kept in the Database Server which is stored in History log from the initial setup of the system to Current time.

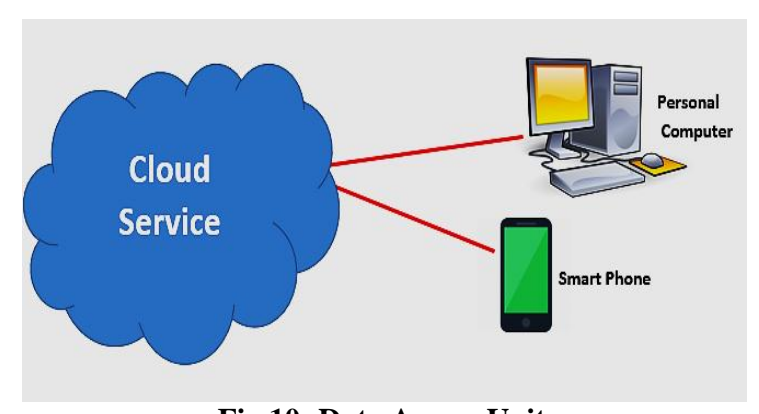

Fig 10: Data Access Unit
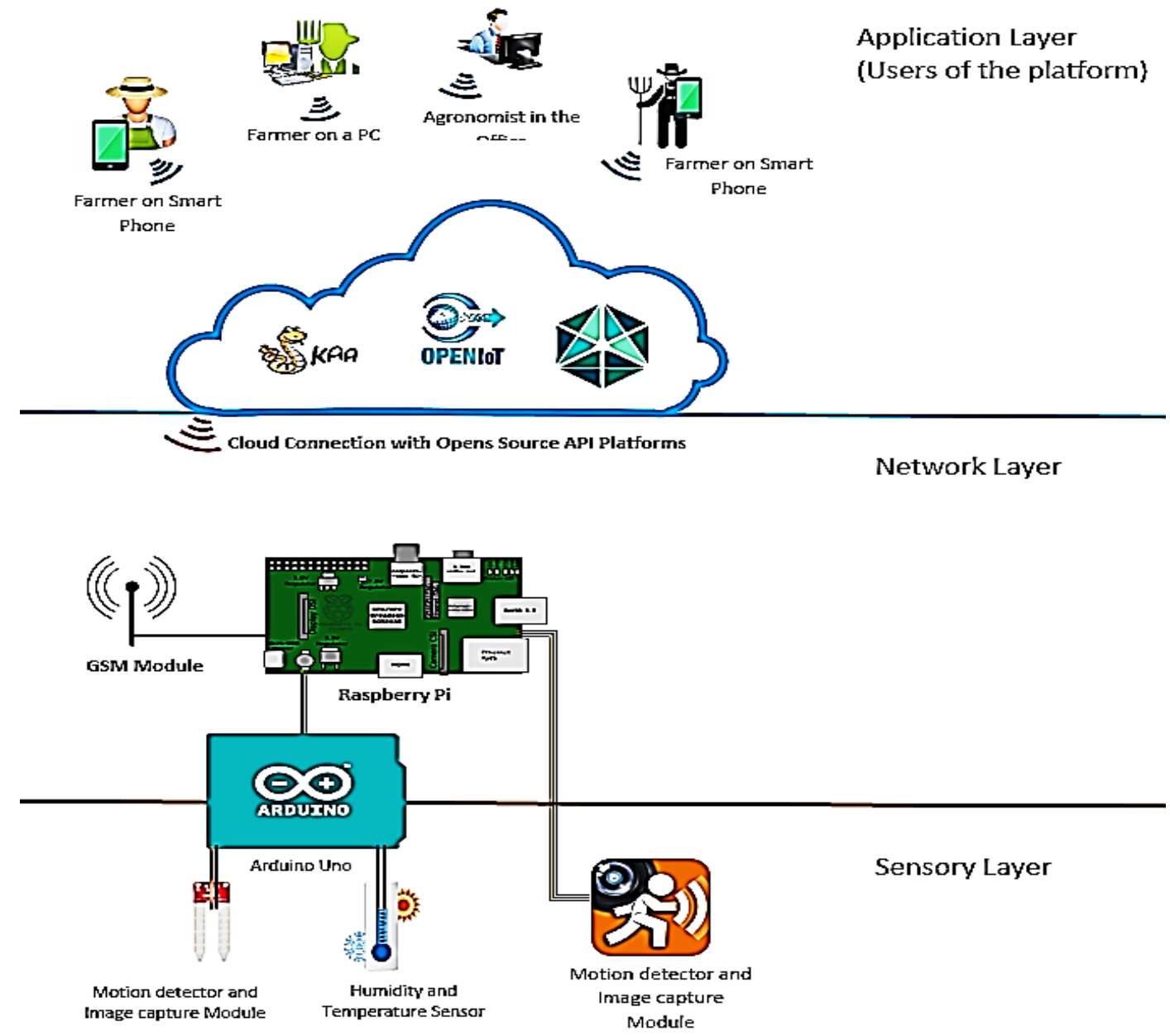

Fig 11: iFarm System Architecture 


\section{SYSTEM IMPLEMENTATION, RESULTS AND ANALYSIS}

\subsection{Implementation}

To ensure a successful, affordable experimentation and prototype built, open source modules of embedded hardware and software systems were used out of which the prototype will also be used to demonstrate how data is sensed or captured and sent to the cloud platform to be assessed by the farmers, agronomist and other stakeholders Three different modules thus Moisture Module, Temperature Module and Motion detection module were tested. The different modules were tested separately for individual or unit testing purposes before the three are integrated and tested as a whole unit or system.

After a successful testing of the three modules, integration is made to have a complete system interlaced with the cloud platform for remote monitoring and control. There are numerous cloud platforms both Open Source and Proprietary that supports and integrates Internet of Things. In this work, the chosen test bed platforms included KAA, Cayenne, Allseen Alliance and OpenIOT which gave tremendous result. Data could be represented in both text and graphical format which makes it very easy to understand and interpret the results at a glance

\subsection{Complete System Prototype}

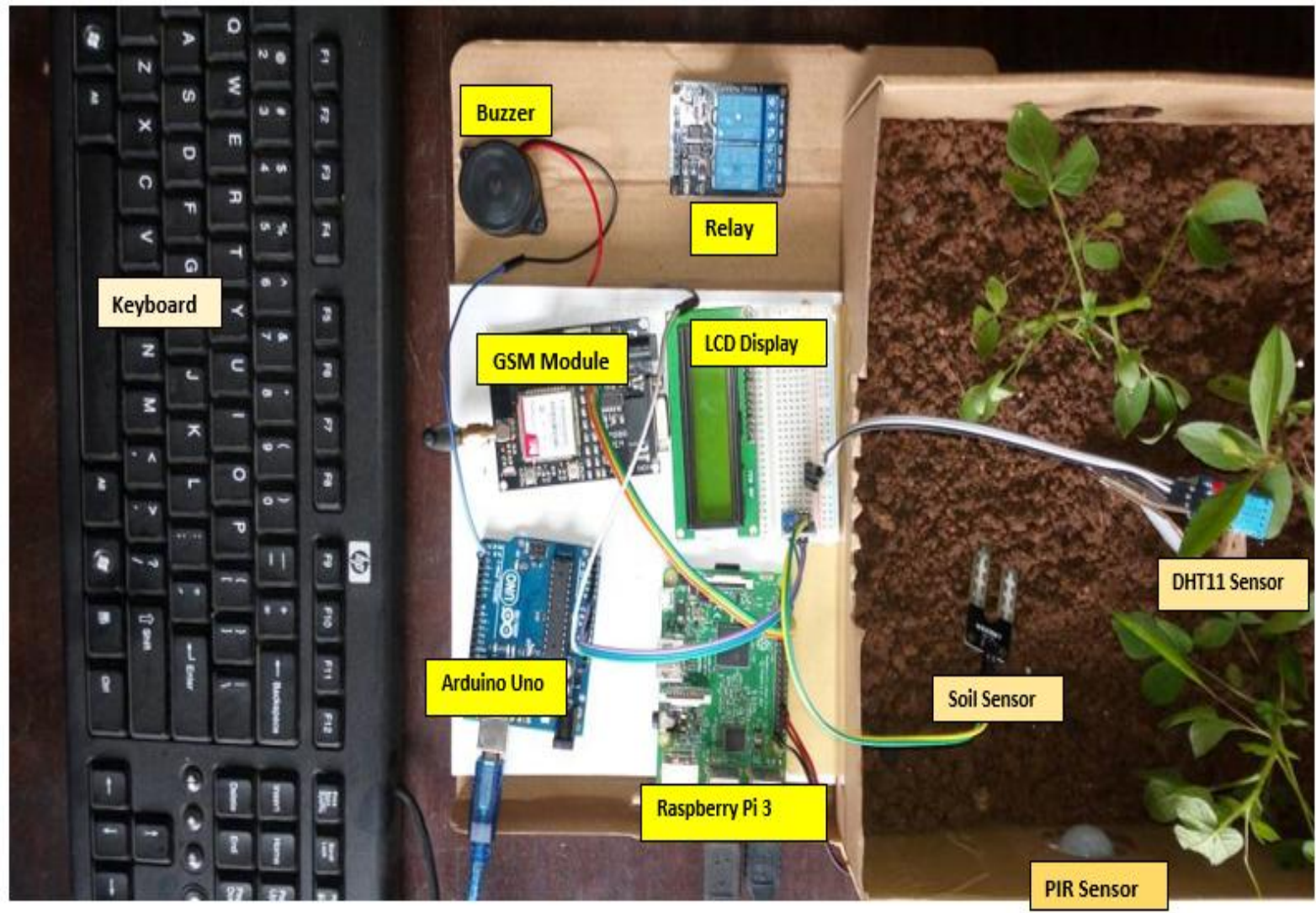

Fig 12: System Prototypes

\subsection{RESULTS AND CONCLUSION}

Upon integrating the three different modules into a system, all the three modules could easily be integrated and the various information expected to be accessed remotely could be achieved at any point in time over existing Open Source APIs.

It can be concluded that with the numerous availabilities of sensors and open source technologies, any kind of information needed by a farmer or agriculturist can easily be obtained remotely with the click of a button or swipe on a smartphone.

\section{REFERENCES}

[1] Aronson, R. L. (2013). FarmBot: Humanity's opensource automated precision farming machine. Retrieved from https://farmbot.io/whitepaper/

[2] Enu, P. (2014). Analysis of the Agricultural Sector of Ghana and Its Economic Impact on Economic Growth, 5(July).
[3] FAO. (2009). FAO - News Article: 2050: A third more mouths to feed. Rome. Retrieved from http://www.fao.org/news/story/en/item/35571/icode/

[4] FAO. (2013). Climate-Smart Agriculture Sourcebook. Sourcebook on Climate-Smart Agriculture, Forestry and Fisheries. Retrieved from http://www.fao.org/docrep/018/i3325e/i3325e00.htm

[5] Farooq, M. U., \& Waseem, M. (2015). A Review on Internet of Things ( IoT ). International Journal of Computer Applications (0975 8887), 113(1), 1-7. https://doi.org/10.5120/19787-1571

[6] Floros, J. D., Newsome, R., Fisher, W., BarbosaC??novas, G. V., Chen, H., Dunne, C. P., ... Ziegler, G. R. (2010). Feeding the world today and tomorrow: The importance of food science and technology. Comprehensive Reviews in Food Science and Food Safety, 9(5), 572-599. https://doi.org/10.1111/j.1541- 


$$
\text { 4337.2010.00127.x }
$$

[7] FuBing. (2015). The Research of Agricultural Expert System based on IOT (pp. 1400-1403). Atlantis Press.

[8] Goldin, I. (2014). FUTURE OPPORTUNITIES , aKey Trends Shaping the Global Economy and Society, (October).

[9] Jayaraman, P. P., \& Palmer, D. (2015). Do-it-Yourself Digital Agriculture Applications with Semantically Enhanced loT Platform. 2015 IEEE Tenth International Conference on Intelligent Sensors, Sensor Networks and Information Processing (ISSNIP) Singapore, 7-9 April 2015, (April), 7-9.

[10] Ma, J., Zhou, X., Li, S., \& Li, Z. (2011). Connecting Agriculture to the Internet of Things through Sensor Networks. 2011 IEEE International Conferences on Internet of Things, and Cyber, Physical and Social Computing. https://doi.org/10.1109/iThings/CPSCom.2011.32

[11] Mohanraj, I., Ashokumar, K., \& Naren, J. (2016). Field Monitoring and Automation using IOT in Agriculture Domain. Procedia - Procedia Computer Science, 93(September), 931-939. https://doi.org/10.1016/j.procs.2016.07.275

[12] O’Halloran, D., \& Kvochko, E. (2015). Industrial Internet of Things: Unleashing the Potential of Connected Products and Services. World Economic
Forum, (January), 40. Retrieved from http://www3.weforum.org/docs/WEFUSA_IndustrialInte rnet_Report2015.pdf

[13]Rankin, D., \& Rankin, D. (2015). Opening Agriculture: Alternative Technological Strategies for Sustainable Farming. Sustainability. Retrieved from http://scholar.colorado.edu/cgi/viewcontent.cgi?article=2 $124 \&$ context=honr_theses

[14] Raul. (2016). IoT Applications With Examples - Internet Of Things Wiki. Retrieved April 26, 2017, from http://internetofthingswiki.com/iot-applicationsexamples/541/

[15] Stringer, R. (2001). How Important are the Nontraditional Economic Roles of Agriculture in Development? (No. 118). Most. South Australia. Retrieved from http://citeseerx.ist.psu.edu/viewdoc/download?doi=10.1. 1.29.3767\&amp;rep=rep1\&amp;type=pdf

[16]Stringer, R., \& Pingali, P. (2004). Agriculture ' s contributions to economic and social development. Electronic Journal of Agricultural and Development Economics, 1(1), 1-5.

[17] The World Bank. (2005). Module 4: Investments in sustainable agricultural intensification. Agriculture investment sourcebook. https://doi.org/10.1596/0-8213$6085-X$ 